\title{
Development and testing of an experimental power system fault demonstrator
}

\author{
C. Rose*, D. W. P. Thomas, M. Sumner, E. Christopher \\ *University of Nottingham, UK, eexcr@nottingham.ac.uk
}

Keywords: Protection; power distribution systems; fault demonstration; fault location; experimental demonstrator.

\begin{abstract}
In this paper a laboratory demonstrator for the study of power system faults is described. The facility has been developed in order to experimentally investigate a number of fault location and power system protection technologies developed by the authors and their colleagues at higher power levels and with more representative system parameters than has previously been possible. In addition to describing the facility itself, this paper also describes the validation of a previously studied method using the new demonstrator.
\end{abstract}

\section{Introduction}

Reliable fault location is desirable in electrical transmission and distribution systems as well as more electric ships and aircraft: automated fault location allows the correct circuits to be isolated while minimising the impact on other parts of the system, and accurate knowledge of where a fault has occurred can substantially reduce repair times [1-3]. In addition, the study of novel equipment and control methods under fault conditions is an important stage of development, as it demonstrates the behaviour of the design under abnormal conditions and can show compliance with relevant grid standards [4-6]. Although it is possible to perform studies on operating power systems - and such studies are of considerable value - results from this type of field testing are not always easily reproduced under controlled conditions and the occurrence of faults and transients is generally unpredictable. As a consequence, fault testing at the early stages of development is often done through simulation or using supplies with limited power availability, as is the case in [7-9].

This paper describes a new power system fault demonstrator, designed specifically to validate a number of fault location algorithms, but also suitable for studying the behaviour of gridconnected equipment under faulted conditions, in a controlled environment where repeatable results may be achieved. Also in this paper, results are presented to validate a fault location method [9] at higher voltage and current levels than has previously been possible. The accuracy of the method is considered and some of the potential sources of error are identified.

\section{The experimental demonstrator}

The power system fault demonstrator is built around a laboratory-based microgrid at the University of Nottingham [10]. The demonstrator may be powered from a number of AC or DC sources in either a grid-connected or isolated mode, and the design is such that the supply impedance may be varied and faults of varying types and severity may be applied at a number of locations. The experimental power system consists of a variable impedance supply connected to a main feeder which links five cabinets, each consisting of the switchgear and protection required to control the loads connected to that cabinet. Each section of the main feeder consists of $10 \mathrm{~m}$ of $16 \mathrm{~mm}^{2}$ cable. A photograph of the experimental facility is shown in Figure 1. The system is illustrated as a one-line diagram in Figure 2.

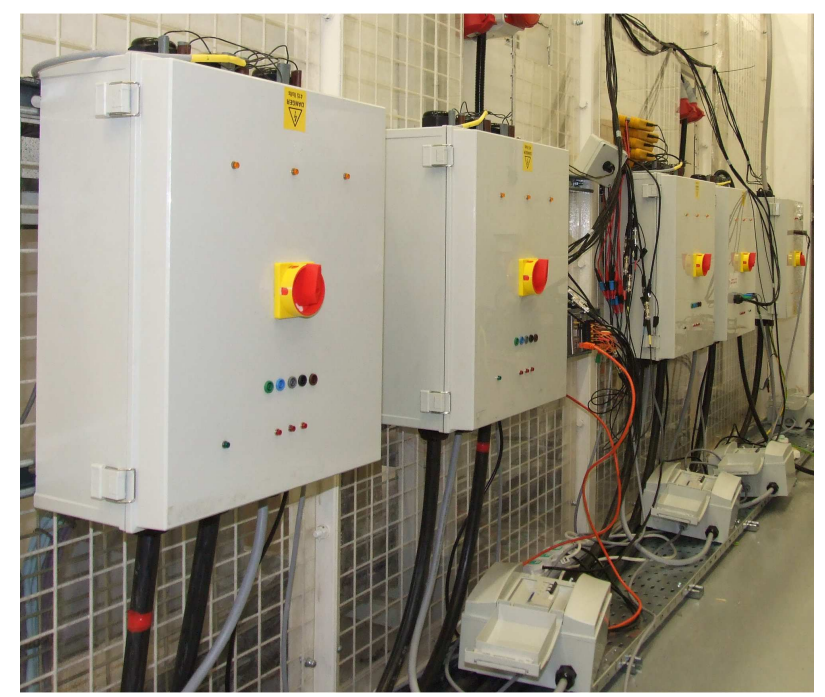

Fig. 1: Photograph of the experimental facility.

The supply is taken from a $300 \mathrm{kVA}$ bussbar fed from a 1 MVA transformer. A number of alternative sources may also be used if desired; connections are available for the supply to be provided by AC or DC power converters or generators while isolated from the grid. The fault current is limited by introducing a variable impedance in series with the supply, consisting 


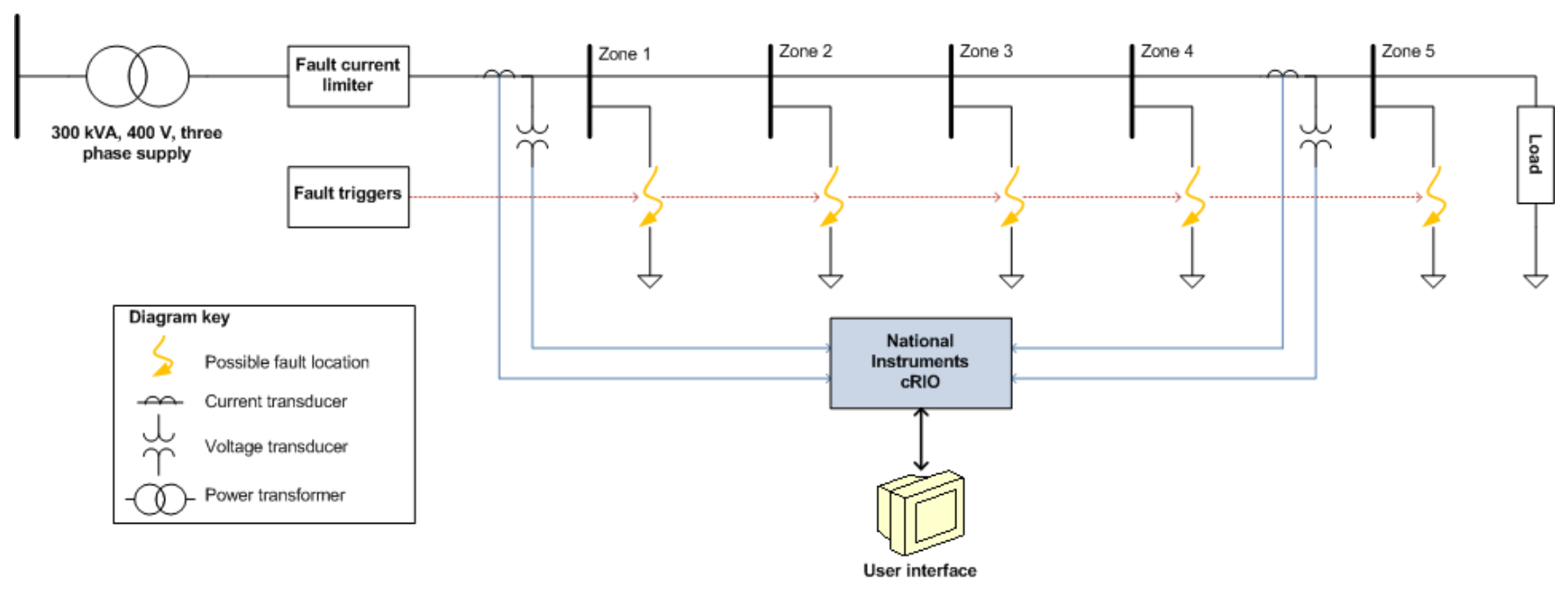

Fig. 2: One line diagram showing the demonstrator layout.

of three-phase inductors with numerous tap positions, allowing the inductance to be varied up to $2 \mathrm{mH}$ and variable highpower resistor banks providing up to $2 \Omega$ of additional supply resistance.

Current and voltage transducers are installed in the first and last cabinets, referred to as Zone 1 and Zone 5 respectively. The installed current transducers are hall effect type sensors with a \pm 300 A input range [11], although the data acquisition hardware will saturate when the measured current magnitude exceeds $250 \mathrm{~A}$. The transducers may be upgraded to allow higher fault currents to be accurately measured. The voltage transducers are differential voltage probes with a $\pm 700 \mathrm{~V}$ input range [12], however, the output of the probes is limited to $70 \%$ of the input range of the sampling hardware. The transducer outputs are sampled using 16-bit analogue to digital converters, giving theoretical resolutions of $0.0038 \mathrm{~A}$ for the current transducers and $0.0153 \mathrm{~V}$ for the voltage transducers, which is a considerably higher resolution than required. At present the maximum allowable fault current is determined by the range of the current transducers.

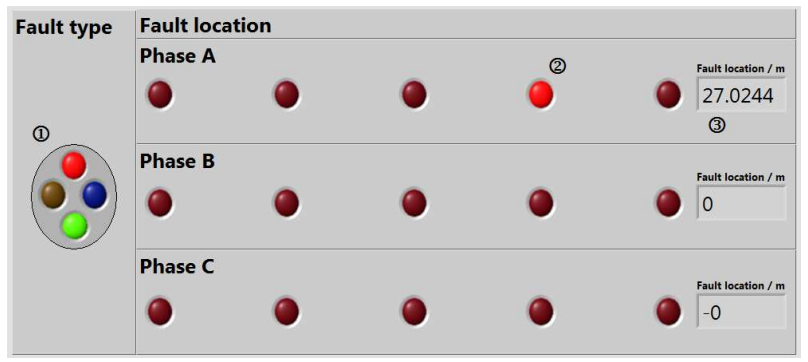

Fig. 3: A screenshot of the user interface showing: (1) the fault type indicator, (2) the fault location indicators, (3) the fault location readout.

Data capture and processing is handled using a National Instruments Compact RIO (cRIO) [13], which contains an FPGA and microprocessor for data capture, processing and control. The cRIO was programmed using LabView. Data acquisition is performed continuously at a sampling frequency of $50 \mathrm{kHz}$. Captured data is then filtered and downsampled to $12.5 \mathrm{kHz}$ using the FPGA. A user interface is provided through a PC. The user interface displays the cRIO output in a human-readable format. Figure 3 shows the important parts of the user interface. The three key features of the interface are the fault type indicator, the fault location indicator and the fault location readout. The fault type indicator shows the type of fault detected by illuminating the indicators associated with the faulted cores of the cable: red, yellow and blue for Line 1, Line 2 and Line 3 respectively and green for the neutral/earth conductor. The fault location indicator gives an immediate, visual indication of which section of cable is faulty, and the location readout gives the estimated distance from the beginning of the line to the fault in metres. The "back-end" of the cRIO software may be configured to implement a number of different fault location methods. In addition to displaying the fault location results, the cRIO also saves the recorded transient data to a file, which may be retrieved using the cRIO's network interface and imported for viewing and analysis using suitable software, such as MATLAB.

In order to impose faults on the system a number of fault units were constructed. The units consist of a contactor connected to the power system on one side and to a miniature circuit breaker (MCB) and a number of shorting links on the other. The fault units can be configured by inserting or removing the shorting links. The MCB ensured eventually disconnection of the fault, even in the event of contactor failure. A fault unit is connected to each of the five cabinets. The faults may be triggered from a control panel consisting of a number of switches, each controlling one of the fault units. Figure 4 shows one of the fault units.

Although the fault units will ideally impose a "bolted" shortcircuit on the system when activated, the transient caused when 


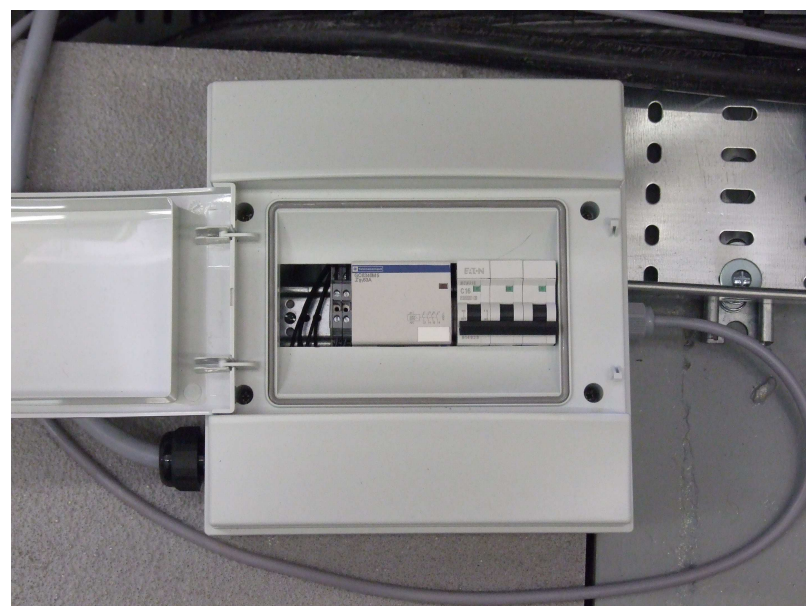

Fig. 4: Photograph of one of the fault units.

the units are first activated is similar to what may be expected from an arc fault; this is caused by the arcing which occurs internally as a result of "switch-bounce" as the contactor closes. This can be seen in Figure 5, which shows a typical fault transient captured by the cRIO, where it takes approximately $2 \mathrm{mS}$ for the voltage to settle after the fault occurs. The effect is also visible to a lesser extent in the current waveform.
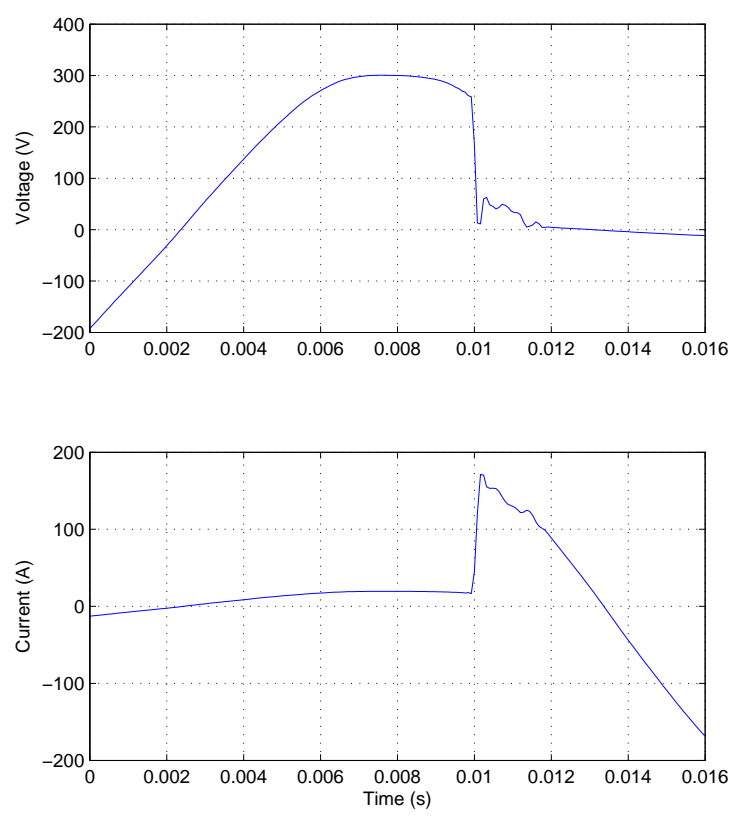

Fig. 5: Typical captured fault transient data.

This section has described the design of the experimental fault demonstrator. Testing of the demonstrator and validation of a fault location algorithm have been carried out and this is described in the next section.

\section{Fault location methodology}

The fault location method used during testing of the facility is a double-ended technique, described in detail in [14]. The method uses current and voltages measurements taken at the sending and receiving ends of the line. Continuous data capture is performed for all transducers as described in the previous section.

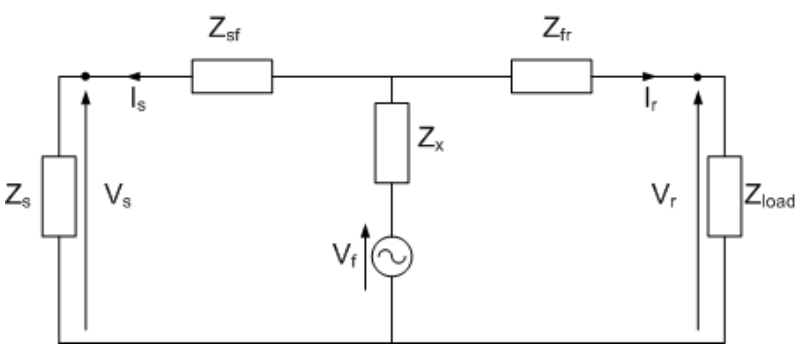

Fig. 6: Equivalent circuit used to derive the fault location algorithm.

When a fault is detected, $5.1 \mathrm{mS}$ of additional data is captured. This is combined to the last $5.1 \mathrm{mS}$ of captured data prior to the fault to give a total of $10.2 \mathrm{mS}$ of data to be analysed. For this work, a basic threshold trigger was used to detect the fault. Captured voltages and currents are preprocessed using a Blackman window to remove the discontinuities at the start and end of the data. An equivalent circuit of a faulted power system is shown in Figure 6. Using Kirchoff's Laws to analyse the equivalent circuit it can be seen that the impedance from the supply to the fault, $Z_{s f}$, may be calculated using (1).

$$
Z_{s f}=\frac{V_{r}-V_{s}+I_{r} Z_{s r}}{I_{s}+I_{r}}
$$

Once the impedance has been estimated at a number of frequencies an ordinary least squares curve fit is applied between $0 \mathrm{~Hz}$ and $3000 \mathrm{~Hz}$. The quality of captured data above $3000 \mathrm{~Hz}$ was found to deteriorate rapidly and was therefore ignored. The resistive part of the results is ignored as it is assumed that the fault will be primarily resistive. The curve-fitted reactance results are then used to calculate the cable inductance between the supply and fault. The distance from the supply to the fault may then be calculated by dividing the estimated inductance by the per unit-length cable inductance.

\begin{tabular}{r|l|l|l} 
Zone & $1 \mathrm{kHz}$ & $2 \mathrm{kHz}$ & $3 \mathrm{kHz}$ \\
\hline 1 & $6.395 \mathrm{uH}$ & $6.130 \mathrm{uH}$ & $5.976 \mathrm{uH}$ \\
2 & $14.66 \mathrm{uH}$ & $13.98 \mathrm{uH}$ & $13.43 \mathrm{uH}$ \\
3 & $21.52 \mathrm{uH}$ & $20.46 \mathrm{uH}$ & $19.69 \mathrm{uH}$ \\
4 & $28.55 \mathrm{uH}$ & $27.17 \mathrm{uH}$ & $26.14 \mathrm{uH}$ \\
5 & $33.86 \mathrm{uH}$ & $32.46 \mathrm{uH}$ & $31.30 \mathrm{uH}$
\end{tabular}

Table 1: Calibrated fault reactance results.

The fault location method studied requires that the total reactance of the line be known or that a reasonable estimate is 

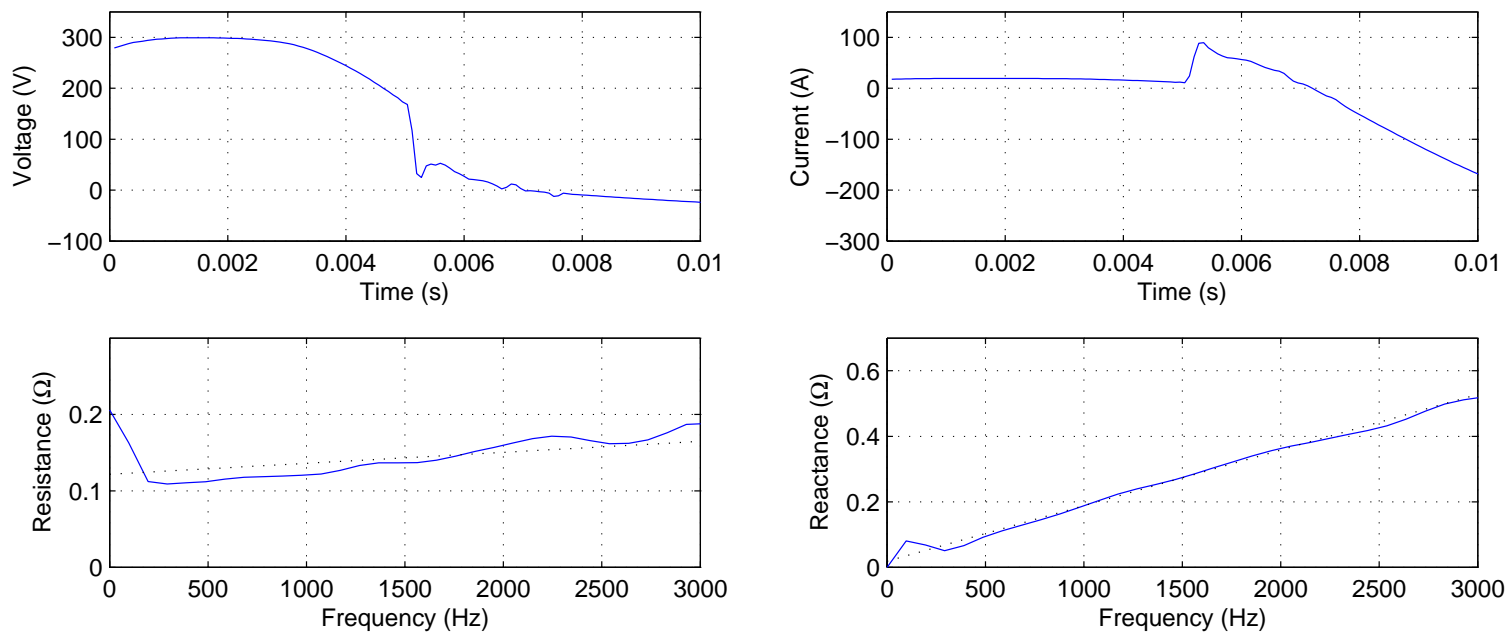

Fig. 7: Full results showing the captured transient supply voltage and current and the estimated impedance for a single-phase to earth fault. The dotted lines on the impedance results are the results after curve fitting.

available. The line inductance was therefore measured using a four-wire impedance analyser for calibration purposes [15]. In addition, the fault inductances were also measured using the impedance analyser. Calibration measurements were taken at $1000 \mathrm{~Hz}, 2000 \mathrm{~Hz}$ and $3000 \mathrm{~Hz}$ for a fault in each of the five zones. Results of the calibration tests are shown in Table 1. The reactance of the whole line is equal to the line reactance seen when a fault occurs in Zone 5 and is therefore not separately listed. Some variation in inductance with frequency is seen. It is not yet clear if this is a measurement error or an actual variation (the cable capacitance is negligible at these frequencies and therefore not the cause). The quality of the fault location results appear to be unaffected by this variation. From the calibration results the cable inductance was found to be approximately $0.82 \mu \mathrm{Hm}^{-1}$. The DC cable resistance was measured and is equal to $3.8 \mathrm{~m} \Omega \mathrm{m}^{-1}$.

\subsection{Experimental results}

Testing of the algorithm was performed a number of times for faults in each location. Individual results were considered in order to identify sources of error and to determine if any improvements to the signal processing could be made. The overall quality of the fault location results was also considered, by analysing the variation in results between tests. The results are presented below.

Figure 7 shows a typical set of results for a single-phase to neutral fault in Zone 5. The fault occurs at approximately $5 \mathrm{mS}$, resulting in a sharp drop in voltage accompanied by a rapid rise in current. The average resistance is $0.143 \Omega$, which is reasonably close to the expected value of $0.152 \Omega$ for a fault in this zone. Using the curve-fitted results for calculation, the inducatance is found to be $30.2 \mu \mathrm{H}$, slightly lower than the expected value of $32.8 \mu \mathrm{H}$, but still within $10 \%$. The fault is then calculated to be $37.3 \mathrm{~m}$ from the supply.
In order to evaluate the overall performance of the algorithm, 40 tests were completed for a phase to neutral fault at each fault location. The mean estimated distance-to-fault, $\overline{\mathrm{D}}$, and the standard deviation, $\sigma$, of the results were calculated using the results for each fault location and used to judge the efficacy of the algorithm. The statistical summary of the results is given in Table 2.

\begin{tabular}{r|l|l} 
Fault & $\overline{\mathrm{D}},(\mathrm{m})$ & $\sigma,(\mathrm{m})$ \\
\hline Zone 1 & -0.84 & 4.05 \\
Zone 2 & 9.48 & 2.91 \\
Zone 3 & 20.2 & 2.94 \\
Zone 4 & 31.1 & 3.83 \\
Zone 5 & 39.9 & 4.88
\end{tabular}

Table 2: Mean estimated distance-to-fault and standard deviations for each zone.

The calculated averages are close to their expected values. From these results it would appear that absolute error in the estimated distance to fault is smallest when the fault occurs near the middle of the line and greatest at the ends of the line. Relative errors are greatest at the supply end of the line and reduce as the fault approaches the load end of the line. The relative errors for results within one standard deviation of the mean in Zones 3, 4 and 5 is already only slightly above $10 \%$. For Zone 2 the error is closer to $20 \%$. The relative error for a fault in Zone 1 is high; this is not unexpected however, since the expected estimated distance for a fault in Zone 1 is very small. Absolute error for Zone 1 is comparable to the absolute errors for Zones 4 and 5.

This section has presented experimental results for a fault location method implemented on the experimental fault demonstrator. In the following section, some of the sources of systematic error in the results have been identified. It is hoped that by 
addressing these errors an overall accuracy of better than $10 \%$ may be consistently achieved using the fault location method described.

\subsection{Sources of error}
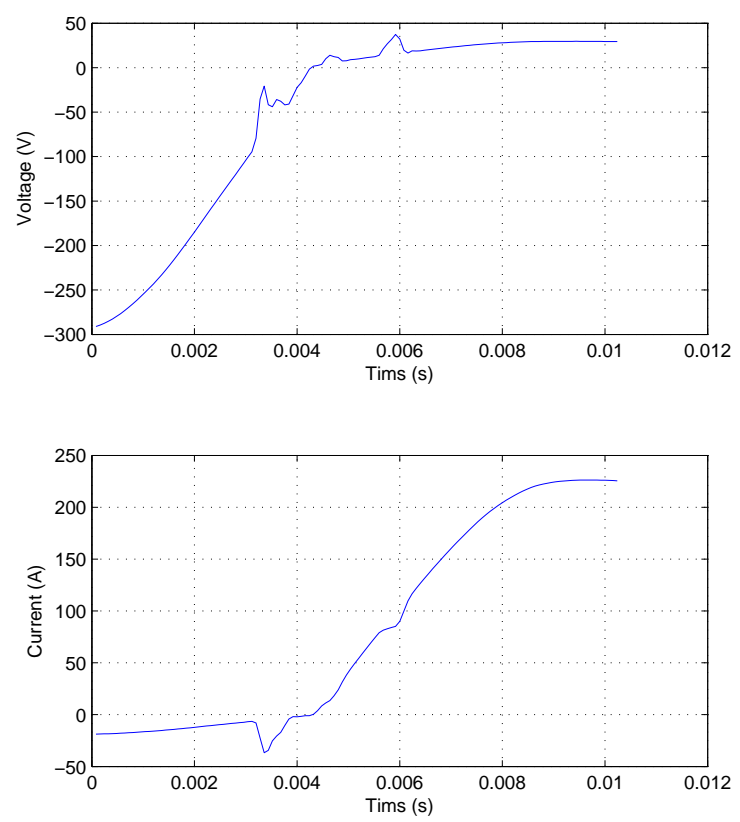

Fig. 8: An example of poorly triggered results. The results should be centred around the fault transient. The transient occurs very close to the voltage zero-crossing, at about $3 \mathrm{mS}$, but is not detected until $5.1 \mathrm{mS}$.

Some of the errors are due to limitations in the fault detection method. The simple threshold trigger which is applied to the measured currents successfully detects all faults; however, if the fault occurs close to the voltage zero crossing, the current will not exceed the threshold until part-way through the next half cycle. As a result, the triggering of the fault location algorithm is delayed and the captured data is not centred around the fault transient. In addition to the delayed triggering, the fault transient is also less significant than for faults occurring near the peak voltage, reducing the amplitude of the transient frequency content used for fault location. This can be seen in Figure 8.

An improved trigger method for the fault location algorithm may improve results but is also likely to be more susceptible to normal system transients. Work is ongoing to find and evaluate a suitable alternative trigger method.

For some results it was found that the fault transients did not contain sufficient frequency content at certain frequencies for accurate reactance estimates to be obtained. This was generally a problem at higher frequencies. The physical cause of this phenomenon is not yet understood. It should be possible to overcome this problem by narrowing the range of frequencies used for the curve fitting. However, an optimum upper frequency limit has not yet been found.

During testing it was found that the choice of MCB used in the fault units has a significant impact on the quality of results. In some cases, it was found that the MCBs in the fault units would trip within a half-cycle of the fault occurring, corrupting the captured data and resulting in unreliable results. The captured fault transient when the MCB tripping current is too low is shown in Figure 9. The early opening of the MCB causes the voltage to recover and the fault current to fall. Early disconnection of the faults is another significant source of error in the results, although obviously not a practical concern with regards to the protection of real systems.

Using MCBs with higher trip currents is the most obvious solution to this problem, although this should be done with care. The MCB trip current must be low enough to ensure disconnection within a few cycles if the fault does not disconnect, but should not be so low as to disconnect the fault immediately. The ratings of the fault unit MCBs have been increased and it is hoped that testing with the revised fault unit design shall be completed soon.
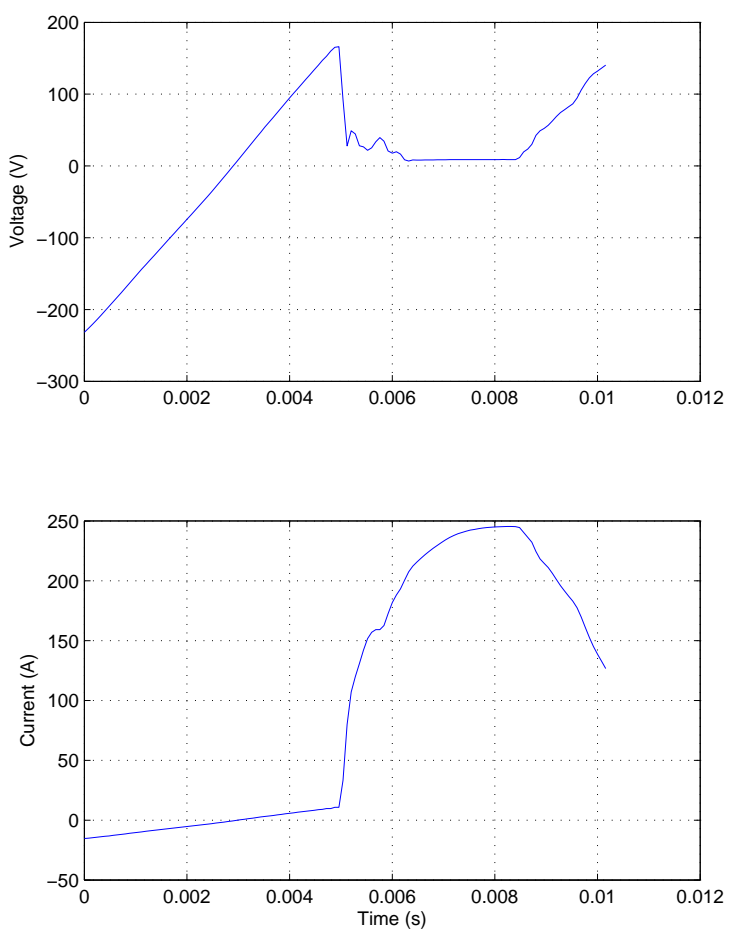

Fig. 9: A captured fault transient, showing the MCB opening at approximately $8 \mathrm{mS}$. 


\section{Conclusions}

This paper has described the design of an experimental fault demonstration facility and the implementation and evaluation of a fault location algorithm. Test results have been presented to demonstrate the method used, which uses relatively low frequency transient voltages and currents to estimate the fault location. Some of the sources of error created by the test procedure have been discussed. Work is ongoing to eliminate or reduce errors where possible. Additional testing is planned to demonstrate the fault location algorithms for DC systems. Testing of the demonstrator with a DC supply is expected to take place in the near future.

Future work is also planned focussing on arc faults. For parallel arc fault studies only relatively minor modifications to the demonstrator are deemed to be necessary as the test methods are similar to those already presented in this report. The authors are also aware of a method of locating series arc faults although some significant modifications to the demonstrator will be necessary before it is possible to study this method further.

\section{Acknowledgements}

The authors would like to thank Nottingham Technology Ventures for funding the construction of the fault demonstrator.

\section{References}

[1] M. M. Saha, J. J. Izykowski and E. Rosolowski, "Fault Location on Power Networks," Springer-Verlag London, 2010

[2] Yanfeng Gong, M. Mynam, A. Guzman, G. Benmouyal and B. Shulim, "Automated fault location system for nonhomogeneous transmission networks," Protective Relay Engineers, 2012 65th Annual Conference for, pp.374381, 2-5 April 2012

[3] Chen Yu, Liu Dong, Xu Bingyin and Huang Yuhui, "Wide area travelling wave fault location in the transmission network," Electricity Distribution (CICED), 2010 China International Conference on, pp.1-6, 13-16 Sept. 2010

[4] S. Loddick, U. Mupambireyi, S. Blair, C. Booth, X. Li, A. Roscoe, K. Daffey, and J. Watson, "The use of real time digital simulation and hardware in the loop to derisk novel control algorithms," Electric Ship Technologies Symposium (ESTS), 2011 IEEE, pp.213-218, 10-13 April 2011

[5] J. Niiranen, "Experiences on voltage dip ride through factory testing of synchronous and doubly fed generator drives," Power Electronics and Applications, 2005 European Conference on,
[6] N. Bottrell and T. C. Green, "Comparison of CurrentLimiting Strategies During Fault Ride-Through of Inverters to Prevent Latch-Up and Wind-Up," Power Electronics, IEEE Transactions on, vol.29, no.7, pp.3786-3797, July 2014

[7] J. Wang, P. Kadanak, M. Sumner, D. W. P. Thomas and R. D. Geertsma, "Active fault protection for an AC zonal marine power system architecture," The 2008 IEEE industry applications society annual meeting (IAS 2008), 5-9 October 2008

[8] Ke Jia, David Thomas and Mark Sumner, "Single-ended fault location scheme for utilization in integrated power system," Power Delivery, IEEE Transactions on, vol.28, no.1, pp.38-46, Jan. 2013

[9] Ke Jia, David Thomas and Mark Sumner, "A New Double-Ended Fault-Location Scheme for Utilization in Integrated Power Systems," Power Delivery, IEEE Transactions on, vol.28, no.2, pp.594-603, April 2013

[10] R. Davies, A. Fazeli, Sung Pil Oe, M. Sumner, M. Johnson and E. Christopher, "Energy management research using emulators of renewable generation and loads," Innovative Smart Grid Technologies (ISGT), 2013 IEEE PES, pp.1-6, 24-27 Feb. 2013

[11] Multicomp, "Current Transducer", datasheet, http://www.farnell.com/datasheets/1368151.pdf

[12] Pico Technology Ltd., "TA041 25MHz +/-700V Differential Probe User's Manual", datasheet, http://www.farnell.com/datasheets/83434.pdf

[13] National Instruments Corporation, "What is NI CompactRIO", webpage, last accessed 23/04/2015, http://www.ni.com/compactrio/whatis/

[14] Ke Jia, "Impedance based fault location in power distribution systems", PhD thesis, University of Nottingham, 2011

[15] Newtons4th Ltd. "LCR Active Head and Impedance Analysis Interface", http://www.newtons4th.com/wpcontent/uploads/2010/02/LCRActiveHead-IAI1.pdf 